\title{
The Apparent Universe
}

\author{
P. Binétruyl1 and A. Helou? \\ AstroParticule et Cosmologie, Université Paris Diderot, CNRS, CEA, \\ Observatoire de Paris, Sorbonne Paris Cité \\ Bâtiment Condorcet, 10, rue Alice Domon et Léonie Duquet, \\ F-75205 Paris Cedex 13, France
}

\begin{abstract}
We exploit the parallel between dynamical black holes and cosmological spacetimes to describe the evolution of Friedmann-Lemaître-RobertsonWalker universes from the point of view of an observer in terms of the dynamics of the apparent horizon. Using the Hayward-Kodama formalism of dynamical black holes, we clarify the role of the Clausius relation to derive the Friedmann equations for a universe, in the spirit of Jacobson's work on the thermodynamics of spacetime. We also show how dynamics at the horizon naturally leads to the quantum-mechanical process of Hawking radiation. We comment on the connection of this work with recent ideas to consider our observable Universe as a Bose-Einstein condensate and on the corresponding role of vacuum energy.
\end{abstract}

\footnotetext{
${ }^{1}$ also at Paris Centre for Cosmological Physics (PCCP),binetruy@apc.univ-paris7.fr.

2alexis.helou@apc.univ-paris7.fr
} 
Since the early proposal of 't Hooft [1] and Susskind [2, the striking parallel between the dynamics of our observable Universe and the dynamics of black holes has been stressed. This has led to the formulation of the holographic principle (see [3] for a review). This has also a deep relation with the AdS/CFT correspondence [4]. On the side of the gravity theory, two seminal works have provided a complementary perspective on the issue. G. Gibbons and S. Hawking [5] have shown that the close connection between event horizons and thermodynamics which has been found in the case of black holes can be extended to cosmological models. And Jacobson [6] proved that the Clausius relation $\delta Q=T d S$, familiar to black hole dynamics, applied to local Rindler horizons allows to recover Einstein's equations.

In this article, we study the connection between these results in the context of Friedmann-Lemaître Robertson-Walker (FLRW) with spherical symmetry. Because cosmology is dynamics, we are using the formalism of Hayward and Kodama [7, 8, which was devised to describe dynamical black holes. As we will see, the notion of apparent horizon, which separates trapped and untrapped surfaces is central to the study.

Our motivation is to identify which part of our classical Universe is relevant for understanding the evolution of the Universe, from our standpoint of observers. This is important if we wish to see this classical Universe as resulting from some measurement process in the quantum mechanical sense. Or more precisely if we want to identify where relevant information accessible to us, as observers, lies in spacetime.

In the next Section, we review the formalism that we will use, borrowed from the study of dynamical black holes, and we apply it to cosmologies of FLRW type. In Section 2, we show how the Clausius relation of thermodynamics applied to the apparent horizon leads to the dynamical evolution of the universe according to the standard laws of FLRW cosmology. In the next Section, we move from thermodynamics to quantum mechanics (in the semi-classical limit) and show how Hawking radiation can be understood in this context. Finally in Section 4, we interpret the results presented.

\section{Black hole dynamics vs cosmic dynamics}

We recall in this Section the formulation of Hayward [7] and Kodama [8] for black hole dynamics, before applying it to cosmology. The major advantage

for us is that this makes the apparent horizon play a natural role (whereas 
the standard approach focuses on the event horizon), which is easily adapted to the cosmological considerations that follow. For simplicity, we consider only spherically symmetric spacetimes: this is general enough to be able to apprehend the dynamics at play in the apparent horizon. We thus consider the following spherically symmetric 4-dimensional metric (with a signature $(-,+,+,+))$ :

$$
d s^{2}=\gamma_{i j}(x) d x^{i} d x^{j}+R^{2}(x) d \Omega^{2},
$$

where the two coordinates $x^{i}\left(x^{0}=t, x^{1}=r\right)$ run over the radial-temporal plane, and $R$ is a function of $t$ and $r$.

In this context, the total gravitational energy enclosed in a sphere of physical radius $R$ is believed to be the Misner-Sharp energy [9]:

$$
E(R) \equiv \frac{R}{2 G}\left(1-\nabla^{a} R \nabla_{a} R\right)
$$

where $G$ is Newton's constant.

A sphere is (anti)trapped, marginal or normal depending on whether $\nabla^{a} R$ is timelike, null or spacelike. Hence, the surface defined by the condition

$$
\nabla^{a} R \nabla_{a} R=0
$$

corresponds to the hypersurface limiting trapped from normal surfaces: it is the apparent horizon, corresponding at a given time to a sphere of radius $R_{A}(t)$ (solution of (3) ). We deduce that the gravitational energy (2) enclosed in the apparent horizon is simply

$$
E\left(R_{A}\right)=\frac{R_{A}}{2 G}
$$

an expression familiar from the Schwarzschild black hole.

In black hole (spherically symmetric) dynamics, one has to replace the classical notion of Killing vector familiar to the stationary black hole case, by the Kodama vector [8]:

$$
K^{a} \equiv \epsilon_{\perp}^{a b} \nabla_{b} R
$$

where $\epsilon_{\perp}^{a b}$ is the $(1+1)$ antisymmetric tensor field in the $(t, r)$ plane. The Kodama vector lies in this plane and is divergence free:

$$
\nabla_{a} K^{a}=0 \text {. }
$$

It satisfies

$$
K^{a} \nabla_{a} R=0, \quad K^{a} K_{a}=-\nabla^{a} R \nabla_{a} R
$$


which allows to express the Misner-Sharp energy (2) in terms of $K^{a} K_{a}$.

A central quantity is the energy flux vector [7]

$$
\psi_{a} \equiv T_{a}^{b} \nabla_{b} R+\omega \nabla_{a} R
$$

where $T_{a b}$ is the energy-momentum tensor and

$$
\omega \equiv-\frac{1}{2} T^{i j} \gamma_{i j}
$$

It measures in particular the departure of the Kodama vector from being a Killing vector (compare with $\nabla_{a} \xi_{b}+\nabla_{b} \xi_{a}=0$ for a Killing vector $\xi_{a}$ )

$$
K^{a}\left(\nabla_{a} K_{b}+\nabla_{b} K_{a}\right)=8 \pi G R \psi_{b}
$$

The Unified First Law of black hole dynamics [7] then reads

$$
\nabla_{a} E=A \psi_{a}+\omega \nabla_{a} V
$$

where $A=4 \pi R^{2}$ and $V=\frac{4 \pi}{3} R^{3}$ are the usual area and volume of the sphere in flat coordinates.

The second term in (11) is interpreted as a work done by the change of the apparent horizon. In order to better understand the first term, and to see in what sense this is the first law of black hole dynamics, we must introduce the surface gravity, which is defined through [7]

$$
K^{a}\left(\nabla_{a} K_{b}-\nabla_{b} K_{a}\right)=2 \kappa \nabla_{b} R
$$

Useful expressions are

$$
\kappa=G \frac{E}{R^{2}}-4 \pi \omega R G
$$

and

$$
\kappa=\frac{1}{2} \operatorname{div} \operatorname{grad} R=\frac{1}{2 \sqrt{-\gamma}} \partial_{i}\left[\sqrt{-\gamma} \gamma^{i j} \nabla_{j} R\right] .
$$

One can then write the first term in the Unified Law as

$$
A \psi_{a}=\frac{\kappa}{8 \pi G} \nabla_{a} A+R \nabla_{a}\left(\frac{E}{R}\right)
$$

Let us now introduce a vector $t^{a}$ tangent to the apparent horizon, which is thus defined by

$$
t^{a} \nabla_{a}\left[\nabla^{b} R \nabla_{b} R\right]=0
$$


Using the definition of Misner-Sharp energy (2), we see that ${ }^{3} t^{a} \nabla_{a}[E / R]=0$. Hence, projecting the Unified First Law tangentially to the apparent horizon, we obtain, using (11) and (15),

$$
d E \equiv t^{a} \nabla_{a} E=\frac{\kappa}{8 \pi G} d A+\omega d V
$$

where $d A=t^{a} \nabla_{a} A$ and $d V=t^{a} \nabla_{a} V$. We recognize in the first term the Clausius relation $\delta Q=T d S$ with the entropy $S$ identified with the area $A$ of the apparent horizon divided by $4 G$, and

$$
T=\frac{\kappa}{2 \pi} .
$$

The first who extended this formalism to cosmology and who stressed the role of the apparent horizon in this context were Bak and Rey in 1999 [10. This was followed by a rather extended literature (see for example [11, 12 and references therein).

From now on, we will focus on Friedmann-Lemaître-Robertson-Walker (FLRW) geometries:

$$
d s^{2}=-d t^{2}+a^{2}(t) \frac{d r^{2}}{1-k r^{2}}+R^{2} d \Omega^{2}, \quad R=r a(t)
$$

Then $\nabla_{t} R=R H$ and $\nabla_{r} R=a$, where $H \equiv \dot{a} / a$ as usual. It follows from (3) that, on the apparent horizon $1-k r^{2}=R^{2} H^{2}$ and thus

$$
R_{A}^{2}=\frac{1}{H^{2}+k / a^{2}}
$$

Also, the Misner-Sharp energy (2) reads

$$
E=\frac{R^{3}}{2 G}\left[H^{2}+\frac{k}{a^{2}}\right]
$$

We will suppose in what follows that the Universe is filled with a (possibly composite) perfect fluid of energy density $\rho$ and pressure $p$. Then the

\footnotetext{
${ }^{3}$ This is often incorrectly stated in the literature by saying that the term $\nabla_{a}(E / R)$ vanishes on the apparent horizon.
} 
Friedmann equation $H^{2}+k / a^{2}=8 \pi G \rho / 3$ leads to the seemingly obvious result

$$
E=\frac{4 \pi}{3} R^{3} \rho
$$

It has been stressed by many authors that it is surprising to see here the flat space volume (even for $k \neq 0$ ): gravitational energy and the perfect fluid energy conspire (together with spherical symmetry) to give this familiar looking contribution.

The Kodama vector has the following components:

$$
K^{t}=\sqrt{1-k r^{2}}, \quad K^{r}=-H r \sqrt{1-k r^{2}} .
$$

One may note that on the apparent horizon $K_{a}=-\nabla_{a} R$. Also in the de Sitter limit ( $k=0$ and $H$ constant), we have $K_{t}=-1$ and $K_{r}=-a H R$ : in other words, the Kodama vector coincides with the dilatational Killing vector $\xi^{a}$.

From (8), one obtains the components of the energy flux vector:

$$
\psi_{t}=-\frac{1}{2}(\rho+p) R H, \quad \psi_{r}=\frac{1}{2}(\rho+p) a .
$$

We note that, in the de Sitter limit $(\rho+p=0), \psi_{a}=0$, in agreement with the fact that, in this limit, one should recover the Killing equation from (10). This seems in contradiction with the role played by $\psi_{a}$ for recovering the Clausius relation (see (17)). We will explain in the next section this apparent contradiction.

Finally, we give the explicit form of the surface gravity (13) or (14):

$$
\kappa=-\frac{R}{2}\left[2 H^{2}+\dot{H}+\frac{k}{a^{2}}\right] .
$$

\section{A closer look at the dynamics at the ap- parent horizon}

As recalled in the Introduction, Jacobson [6, 13] has shown that one recovers Einstein equations from the Clausius relation applied to a local Rindler horizon. More precisely, the fraction rate of increase of the area element of a 
local Rindler horizon with respect to the affine parameter $\lambda$ is the expansion $\theta$ of the null congruence of horizon generators:

$$
\delta A=\int \theta d \lambda d A
$$

where $\lambda$ is the affine parameter. The geodesic deviation equation gives the Raychaudhuri equation for the expansion (see for example [14], chapter 9):

$$
\frac{d \theta}{d \lambda}=-\frac{1}{2} \theta^{2}-\sigma^{2}-R_{a b} k^{a} k^{b}
$$

where $\sigma^{2}=\sigma_{a b} \sigma^{a b}$ is the squared shear of the congruence, $R_{a b}$ the Ricci tensor and $k^{a}$ is the (null) affine tangent vector to the congruence. At the local Rindler horizon, $\theta^{2}$ and $\sigma^{2}$ vanish; thus $\theta=-\lambda R_{a b} k^{a} k^{b}$ and

$$
\delta A=-\left.\int \lambda R_{a b} k^{a} k^{b} d \lambda d A\right|_{\mathcal{H}} .
$$

The Clausius relation $\delta Q=T d S$, with $T=\kappa /(2 \pi)$ and $d S=\eta \delta A(\eta$ constant to be determined) thus reads:

$$
\delta Q=-\left.\frac{\kappa}{2 \pi} \int \eta \lambda R_{a b} k^{a} k^{b} d \lambda d A\right|_{\mathcal{H}} .
$$

On the other hand, introducing the local boost Killing vector $\xi^{a}$, one may write the energy flux through the element of horizon surface $d \Sigma^{a}=k^{a} d \lambda d A$ as

$$
\delta Q=\int T_{a b} \xi^{a} d \Sigma^{b}=-\left.\kappa \lambda \int T_{a b} k^{a} k^{b} d \lambda d A\right|_{\mathcal{H}},
$$

where we used $\xi^{a}=-\kappa \lambda k^{a}$. Comparing (29) and (30) we obtain $T_{a b} k^{a} k^{b}=$ $\eta R_{a b} k^{a} k^{b} /(2 \pi)$ for all null $k^{a}$, which implies $(2 \pi / \eta) T_{a b}=R_{a b}+f g_{a b}$ for some function $f$, which is easily determined to be $f=-R^{a}{ }_{a}+\Lambda$. One thus recovers Einstein's equations with a cosmological constant $\Lambda$, for $\eta=1 /(4 G)$.

It is essential in Jacobson's derivation to consider the locus of vanishing expansion (in his case the local Rindler horizon, since it is instantaneously stationary). At a more global level, this is precisely the definition of the apparent horizon. One may thus expect that the Clausius relation applied to the apparent horizon allows to recover the dynamics of FLRW. This is what we will now prove, using the formalism introduced in the previous section. 
In particular, as we will see, when going from local Rindler horizon to the apparent horizon, the Killing vector is replaced by the Kodama vector.

In order to describe the appropriate null geodesic congruences, we define the null vectors

$$
\begin{aligned}
k^{a} & \equiv \frac{1}{2}\left(1,-\frac{\sqrt{1-k r^{2}}}{a}, 0,0\right), \\
l^{a} & \equiv 2\left(1, \frac{\sqrt{1-k r^{2}}}{a}, 0,0\right) .
\end{aligned}
$$

The vector $k^{a}$, tangent to the light rays, is chosen to describe the futureoriented ingoing null congruence. And the vector $l^{a}$ is introduced in such a way that $k^{a} l_{a}=-2$. In this way, the metric [3]

$$
h_{a b} \equiv g_{a b}+\frac{1}{2}\left(k_{a} l_{b}+k_{b} l_{a}\right)
$$

is the induced metric on the 2-spheres (at fixed $r$ and $t$ ). Then, the expansion is defined as [14]

$$
\theta=h^{c d} \nabla_{c} k_{d}
$$

where $h^{c d}$ is the inverse induced metric. It follows that the expansion for the future-directed ingoing null geodesic congruence is simply [10]

$$
\theta=H-\frac{1}{R} \sqrt{1-k r^{2}}
$$

We check that the apparent radius (20) corresponds to vanishing expansion. We also note that, on the apparent horizon, the vector $k^{a}$ just introduced is aligned with the Kodama vector $K^{a}$ in (23) (and thus with $\nabla^{a} R$ as well):

$$
\left.k_{a}\right|_{\mathcal{H}}=\frac{1}{2}\left(-1,-\frac{a}{H R}, 0,0\right)=\left.\frac{1}{2 H R_{A}} K_{a}\right|_{\mathcal{H}}
$$

As emphasized above, the apparent radius is time-dependent: we have from (20)

$$
\frac{2 \dot{R}_{A}}{H R_{A}}=2\left(1-\frac{H^{2}+\dot{H}}{H^{2}+k / a^{2}}\right) \equiv \beta^{2} .
$$

The notation $\beta$ is motivated by the fact that, in the (A)dS/CFT correspondence, this is nothing but the beta function of the associated renormalisation 
group flow, as we will discuss below4. We note that the surface gravity (25) simply reads

$$
\kappa=-\frac{R}{R_{A}^{2}}\left(1-\frac{\beta^{2}}{4}\right)
$$

Finally, we have introduced in (16) the vector $t^{a}$ tangent to the apparent horizon, which satisfies $t^{a} \nabla_{a}[E / R]=0$. One easily checks that, up to a normalization factor,

$$
\begin{aligned}
t^{a} & =\frac{1}{2}\left(1,-\frac{R H}{a}\left(1-\beta^{2} / 2\right), 0,0\right) \\
& =\left(1-\frac{\beta^{2}}{4}\right) k^{a}+\frac{\beta^{2}}{16} l^{a} .
\end{aligned}
$$

We have seen above that the Clausius relation is obtained directly from projecting the Unified First Law tangentially to the apparent horizon. Since we have, on the apparent horizon, $l^{a} \psi_{a}=0$ as well as the identification (36), we obtain from (8)

$$
\begin{aligned}
\left.t^{a} \psi_{a}\right|_{\mathcal{H}} & =\left.\left(1-\beta^{2} / 4\right) k^{a} \psi_{a}\right|_{\mathcal{H}}=-\left.\kappa R_{A} k^{a} \psi_{a}\right|_{\mathcal{H}}=-\left.\frac{\kappa}{2 H} K^{a} \psi_{a}\right|_{\mathcal{H}} \\
& =\left.\frac{\kappa}{2 H} T_{a b} K^{a} K^{b}\right|_{\mathcal{H}},
\end{aligned}
$$

where we have used (38) as well as the fact that, on the apparent horizon, $K_{a}=-\nabla_{a} R$ (both of them being null). We note the similarity with (30) where the role of the Killing vector is now played by the Kodama vector.

We thus obtain $\left(A=4 \pi R^{2}\right)$

$$
\left.A t^{a} \psi_{a}\right|_{\mathcal{H}}=2 \pi \kappa H R_{A}^{4}(p+\rho) .
$$

whereas one gets from (15):

$$
\left.A t^{a} \psi_{a}\right|_{\mathcal{H}}=\left.\frac{\kappa}{8 \pi G} t^{a} \nabla_{a} A\right|_{\mathcal{H}}=\left.\frac{\kappa R_{A}}{G} t^{a} \nabla_{a} R\right|_{\mathcal{H}}=\frac{1}{4 G} \beta^{2} \kappa H R_{A}^{2}
$$

Hence

$$
\beta^{2}=8 \pi G R_{A}^{2}(p+\rho),
$$

\footnotetext{
${ }^{4}$ Note that de Sitter i.e. $k=0$ and $H$ constant, corresponds to a zero of the beta function.
} 
or equivalently, using (37),

$$
\dot{H}-\frac{k}{a^{2}}=-4 \pi G(p+\rho) .
$$

We have thus recovered the dynamics of FLRW universes from the Clausius relation.

We understand why the flux vector $\psi_{a}$ vanishes for a de Sitter spacetime $(p+\rho=0)$ : in that case there is no dynamics at the apparent horizon. We also see that the dynamics at the apparent horizon is fully described by the holographic beta function.

\section{Hawking radiation at the apparent horizon}

The preceding considerations have been purely thermodynamical. We now proceed to consider the quantum mechanical process of Hawking radiation [18. Hawking radiation at the apparent horizon has been considered by many authors, both for dynamical black holes or cosmological universes [11, 15, 16, 19. We will follow here the Hamilton-Jacobi formulation of the PauliWilczek tunneling method [17], more precisely the work of Ref.[18] on black holes.

We start by rewriting the metric (19) in terms of the retarded $u$ and advanced $v$ conformal time coordinates:

$$
\begin{aligned}
d s^{2}=-a^{2} & \left(\frac{u+v}{2}\right) d u d v+R^{2} d \Omega^{2} \\
u & \equiv \eta-\chi, \quad v \equiv \eta+\chi \quad, \quad d \eta \equiv \frac{d t}{a(t)}, d \chi \equiv \frac{d r}{\sqrt{1-k r^{2}}} .
\end{aligned}
$$

We then move to the outgoing Eddington-Finkelstein coordinates where the metric takes the following form:

$$
d s^{2}=-e^{2 \Psi} C d U^{2}-2 e^{\Psi} d U d R+R^{2} d \Omega^{2},
$$

with $(d u=d U)$

$$
e^{2 \Psi} C=a^{2} \partial_{U} v, \quad 2 e^{\Psi}=a^{2} \partial_{R} v,
$$

which requires $\partial_{R} v>0$. We have more explicitly

$$
\begin{aligned}
C & =1-k r^{2}-R^{2} H^{2}=1-\frac{2 G E}{R}, \\
e^{\Psi} & =\frac{a}{\sqrt{1-k r^{2}}+R H} .
\end{aligned}
$$


Since $U$ is a retarded time, any trapped $(C<0)$ or marginal $(C=0)$ surface is past trapped or marginal, as expected in our cosmological set up.

The Kodama vector is simply $K=e^{-\Psi} \partial_{U}$ and the surface gravity (14) simply reads

$$
\kappa=\frac{1}{2}\left(\partial_{R} C+C \partial_{R} \Psi\right)
$$

We see that, close to the apparent horizon, where $C \sim 0$, we have $\kappa \sim \partial_{R} C / 2$.

The BKW approximation of the tunneling probability along the classically forbidden trajectory from outside the horizon to inside takes the form 5 :

$$
\Gamma \propto \exp \left(-2 \frac{\operatorname{Im} I}{\hbar}\right)
$$

where $\operatorname{Im} I$ is the imaginary part of the action $I$ on the classical trajectory, to leading order in $\hbar$.

We consider a massless scalar field $\phi \equiv \phi_{0} \exp (i I)$. The equation of motion is the Hamilton-Jacobi equation:

$$
g^{a b} \nabla_{a} I \nabla_{b} I=0 .
$$

The classical action $I$ simply reads

$$
I=-\int \omega e^{\Psi} d U+\int k d R
$$

where we have introduced the Kodama energy $\omega \equiv-K^{a} \nabla_{a} I=-e^{-\Psi} \partial_{U} I$ and $k \equiv \partial_{R} I$. Then, from (53), one obtains

$$
k(C k+2 \omega)=0 .
$$

The solution $k=0$ corresponds to outgoing solutions, whereas $k=-2 \omega / C$ corresponds to ingoing solutions. Near the apparent horizon, $C \sim(R-$ $\left.R_{A}\right) \partial_{R} C \sim 2 \kappa\left(R-R_{A}\right)$. Hence the ingoing solution contributes to the imaginary part of the action

$$
\operatorname{Im} I=\operatorname{Im} \int \frac{-\omega}{\kappa\left(R-R_{A}-i 0\right)} d R=-\frac{\pi \omega}{\kappa}
$$

\footnotetext{
${ }^{5}$ We restore $\hbar$ solely for this equation but set it to 1 everywhere else.

${ }^{6}$ One easily shows that, on the apparent horizon, $k \propto \partial_{\eta} I+\partial_{\chi} I$. Hence $k=0$ corresponds to $I(\eta-\chi)$ i.e. outgoing solutions.
} 
The probability (52) takes the thermal form $\gamma=\exp (-\omega / T)$ with the temperature

$$
T=-\frac{\kappa}{2 \pi} .
$$

Note the difference of sign with respect to (18), which is consistent with the fact that, in the cosmological set up, the surface gravity $\kappa$ is negative (for $p<\rho / 3$, see (38) $)$ [19].

We see that, contrary to the black hole case, the relevant modes are ingoing, which is compatible with the fact that the observer is not at infinity, as in the black hole, but at the center $(r=0)$ : Hawking radiation is aimed at this central observer.

It is informative also to work with Kodama time [12]. Indeed, introducing the Kodama time $\tau$ by writing

$$
d \tau=d U+\frac{e^{-\Psi}}{C} d R
$$

the metric (47) simply reads:

$$
d s^{2}=-e^{2 \Psi} C d \tau^{2}+\frac{1}{C} d R^{2}+R^{2} d \Omega^{2} .
$$

In this metric, the Kodama vector is simply $K=e^{-\Psi} \partial_{\tau}$. A fiducial observer has a 4 -velocity parallel to the Kodama vector $U^{a}=\left(e^{-\Psi} / \sqrt{C}, 0,0,0\right)$. The only non-vanishing component of its acceleration $A^{a}=U^{b} \nabla_{b} U^{a}$ is $A^{1}=$ $C \partial_{R} \Psi+\frac{1}{2} \partial_{R} C$. Hence defining $A^{2} \equiv A^{a} A_{a}$, we have

$$
A=\frac{1}{\sqrt{C}}\left(C \partial_{R} \Psi+\frac{1}{2} \partial_{R} C\right)
$$

The Unruh temperature measured at $R$ is then given by the Kodama energy $e^{-\Psi} A /(2 \pi)$ :

$$
T_{U}(R)=e^{-\Psi(R)} \frac{1}{2 \pi \sqrt{C(R)}}\left|C \partial_{R} \Psi+\frac{1}{2} \partial_{R} C\right|(R),
$$

and the temperature measured by an observer at the origin is obtained by multiplying by the redshift factor $\left|g_{\tau \tau}\right|^{1 / 2}$ :

$$
T_{U, 0}(R)=\frac{\left|g_{\tau \tau}\right|^{1 / 2}(R)}{\left|g_{\tau \tau}\right|^{1 / 2}(0)} T_{U}(R)=\frac{1}{2 \pi}\left|C \partial_{R} \Psi+\frac{1}{2} \partial_{R} C\right|(R),
$$


In particular, one recovers the Hawking temperature (57) by going to the apparent horizon where $C=0$ :

$$
T=T_{U, 0}\left(R=R_{A}\right)=\frac{1}{4 \pi}\left|\partial_{R} C\right|_{\mathcal{H}}=\frac{|\kappa|}{2 \pi} .
$$

\section{The quantum portrait of our visible Uni- verse}

We conclude by proposing an interpretation of the results above. We have seen that a genuine dynamics for the evolution of the Universe appears only when one departs from the de Sitter regime. Indeed, the formalism that we have used has allowed us to make a precise description of this departure in terms of horizon dynamics. The beta function $\beta^{2}=2 \dot{R}_{A} /\left(H R_{A}\right)$ (see (37)) is non-vanishing, due to the energy content of the Universe: as we have seen in (43) $\beta^{2}=8 \pi G R_{A}^{2}(p+\rho)$. We note that all forms of energy besides vacuum energy generate a nonzero beta function. This function is nothing but the holographic beta function introduced in the context of AdS/CFT correspondence [4]: it quantifies the renormalisation group flow associated with departures from conformal symmetry. On the gravitational side, this means that there is no arrow of time in the context of de Sitter geometry (a fixed point of the beta function). The arrow of time is induced by forms of energy other than vacuum energy.

In [20, we promoted the idea that the classical Universe that we observe results from a projection of the quantum state of the Universe through the measurement process. Since time is intrinsically associated with this measurement process, this implies a departure from conformal symmetry, or in other words a nonzero beta function. Our results presented in this paper indicate that the classical Universe that we should consider is the spacetime region bounded by the apparent horizon. We showed in [20], using a model of the classical Universe as a Bose-Einstein condensate (in analogy with black holes [21]), that such a region filled with vacuum energy corresponds to maximal entropy, and thus maximal probability. Again, the process of measurement leads to a departure from such a de Sitter regime. The renormalization group flow will eventually lead to another fixed point and a return to the de Sitter regime.

One may be surprised to find that the apparent horizon plays a central role in this picture: we are presently seeing galaxies and stellar objects such 
as GRBs which are beyond the apparent horizon (but within our particle horizon). Let us first note that a key assumption in our analysis is spherical symmetry. We must thus consider a spherical shell that lies just outside the apparent horizon and enters it during the time $\delta t$ that we make observations. This is represented in Figure 1, in the example of a spatially flat Universe. In this case, the apparent horizon is simply the Hubble horizon and includes all points that recede from the central observer at a speed smaller than the speed of light. Outside the horizon, because of the expansion, photons emitted towards the observer recede from him (in proper distance) until they are overcome by the apparent horizon: from then onwards, they move closer to the observer. If observation lasts $\delta t$, an infinitesimal section of spacetime (limited by the two corresponding light cones, as seen from Figure 1) moves into the apparent horizon. The corresponding information is encoded into the Hawking radiation that reaches the observer.

This work may be pursued along several lines. First, one may relax the assumption of spherical symmetry in order to be able to encode non-spherical fluctuations. Second, the picture that emerges shows some definite differences with the leaking Bose-Einstein model of black hole proposed by Dvali and Gomez [21, 22] because Hawking radiation leaks into the observable patch of the Universe.

Finally, the fertile parallel between cosmological Universe and black hole may in return be an inspiration to understand the dynamics of nonstatic black holes, in particular the presence or absence of event horizon [23, 24].

Acknowlegments: We acknowledge the financial support of the UnivEarthS Labex program at Sorbonne Paris Cité (ANR-10-LABX-0023 and ANR-11-IDEX-0005-02). We wish to thank T. Jacobson, E. Kiritsis, S. Mukhanov and G. Veneziano for useful conversations.

\section{References}

[1] G. 't Hooft, gr-qc/9310026.

[2] L. Susskind, J. Math. Phys. 36 (1995) 6377 hep-th/9409089.

[3] R. Bousso, Rev. Mod. Phys. 74 (2002) 825 hep-th/0203101].

[4] J. M. Maldacena, Adv. Theor. Math. Phys. 2 (1998) 231 hep-th/9711200]. 
[5] G. W. Gibbons and S. W. Hawking, Phys. Rev. D 15 (1977) 2738.

[6] T. Jacobson, Phys. Rev. Lett. 75 (1995) 1260 [gr-qc/9504004.

[7] S. A. Hayward, Class. Quant. Grav. 15 (1998) 3147 [gr-qc/9710089].

[8] H. Kodama, Prog. Theor. Phys. 63 (1980) 1217.

[9] C. W. Misner and D. H. Sharp, Phys. Rev. 136 (1964) B571.

[10] D. Bak and S. -J. Rey, Class. Quant. Grav. 17 (2000) L83 hep-th/9902173.

[11] R. -G. Cai and L. -M. Cao, Phys. Rev. D 75 (2007) 064008 gr-qc/0611071.

[12] G. Abreu and M. Visser, Phys. Rev. D $82 \quad$ (2010) 044027 arXiv:1004.1456 [gr-qc]].

[13] T. Jacobson and R. Parentani, Found. Phys. 33 (2003) 323 gr-qc/0302099].

[14] R. M. Wald, "General Relativity,” Chicago, Usa: Univ. Pr. ( 1984) 491p

[15] R. -G. Cai, L. -M. Cao and Y. -P. Hu, Class. Quant. Grav. 26 (2009) 155018 [arXiv:0809.1554 [hep-th]].

[16] T. Zhu and J. -R. Ren, Eur. Phys. J. C 62 (2009) 413 arXiv:0811.4074 [hep-th]].

[17] M. K. Parikh and F. Wilczek, Phys. Rev. Lett. 85 (2000) 5042 hep-th/9907001.

[18] S. A. Hayward, R. Di Criscienzo, L. Vanzo, M. Nadalini and S. Zerbini, Class. Quant. Grav. 26 (2009) 062001 [arXiv:0806.0014 [gr-qc]].

[19] R. Di Criscienzo, S. A. Hayward, M. Nadalini, L. Vanzo and S. Zerbini, Class. Quant. Grav. 27 (2010) 015006.

[20] P. Binetruy, arXiv:1208.4645 [gr-qc].

[21] G. Dvali and C. Gomez, Fortsch. Phys. 61 (2013) 742 arXiv:1112.3359 [hep-th]]. 
[22] G. Dvali and C. Gomez, arXiv:1312.4795 [hep-th].

[23] S. W. Hawking, arXiv:1401.5761 [hep-th].

[24] A. Almheiri, D. Marolf, J. Polchinski and J. Sully, JHEP 1302 (2013) 062 arXiv:1207.3123 [hep-th]]. 


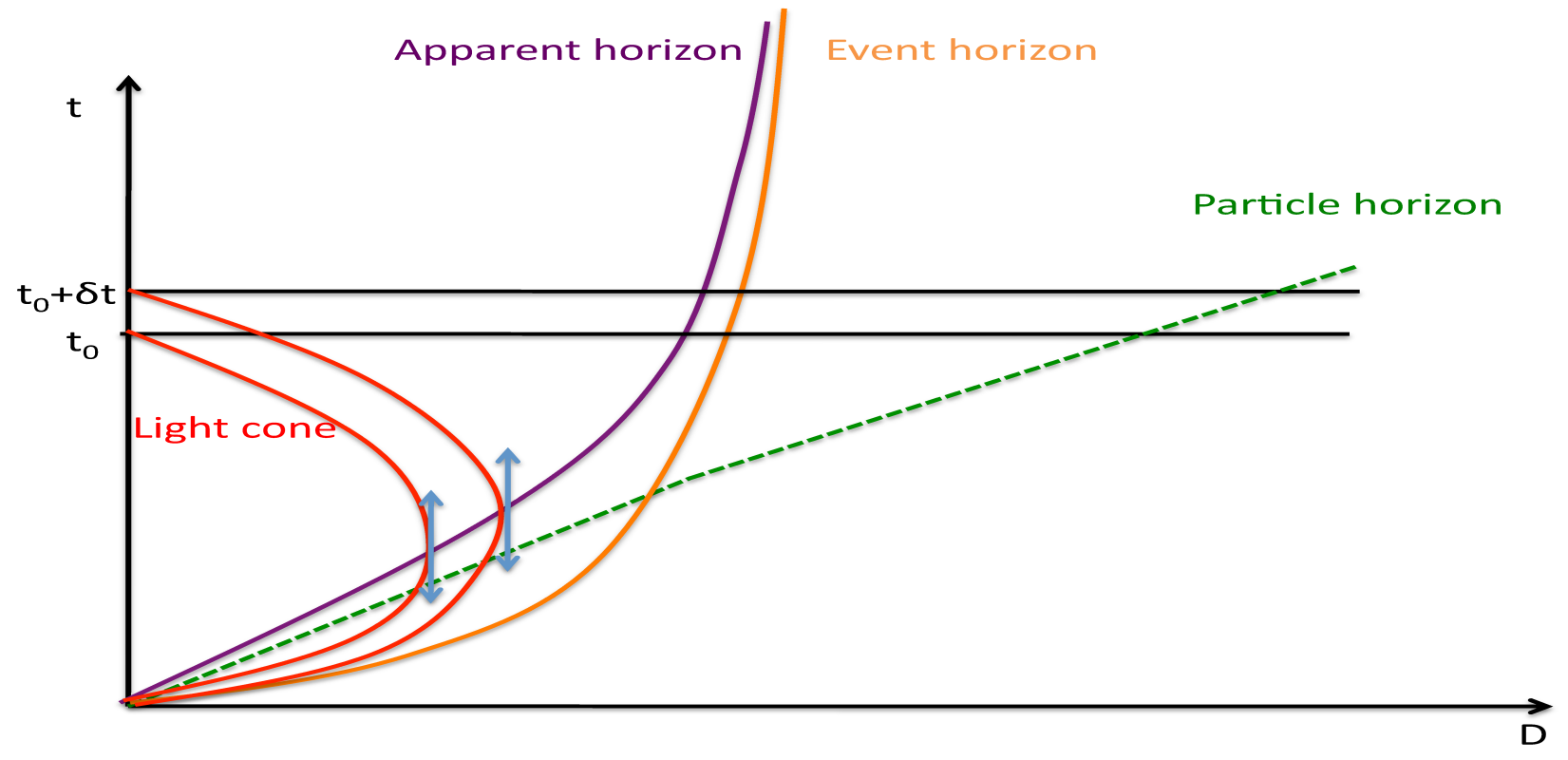

Figure 1: The different horizons (apparent in purple, event in orange, particle in green) as well as the light cones (in red) for an observer at $t_{0}$ and $t_{0}+\delta t$, represented in the plane time versus proper distance, for a Universe with $70 \%$ vacuum energy and 30\% non-relativistic matter. 